\title{
Um Framework Conceitual para Recomendação de Estratégias de Aprendizagem utilizando Motivação e Trilhas de Aprendizagem
}

\section{David Brito Ramos ${ }^{1,2}$, Ilmara Monteverde Martins Ramos ${ }^{2}$, Isabela Gasparini ${ }^{3}$, Elaine Harada Teixeira de Oliveira ${ }^{1}$}

${ }^{1}$ Instituto de Computação - Universidade Federal do Amazonas (UFAM) - Manaus AM - Brasil

${ }^{2}$ Instituto Federal de Educação, Ciência e Tecnologia do Amazonas (IFAM) - Campus Parintins - Parintins - AM - Brasil

${ }^{3}$ Depto de Ciência da Computação - Universidade do Estado de Santa Catarina (UDESC) - Joinville - SC - Brasil

\{david.brito,ilmara.martins\}@ifam.edu.br, isabela.gasparini@udesc.br, elainedicomp.ufam.edu.br

\begin{abstract}
This paper describes a conceptual framework for learning strategies recommendation using the achievement goals theory and learning paths. The framework consists of the student model, learning strategies, classification module, and recommendation module. The student views the recommendations and evaluates them. These ratings make it possible to assess whether the recommended strategy is significant for students and, in parallel, whether it is correlated with their motivations. To date, students have given good ratings to the recommended strategies.
\end{abstract}

Resumo. Este artigo descreve um framework conceitual para a recomendação de estratégias de aprendizagem utilizando a Teoria de Metas de Realização e trilhas de aprendizagem. O framework é composto pelo modelo do estudante, estratégias de aprendizagem, módulo de classificação e módulo de recomendação. $O$ estudante visualiza as recomendações e as avalia. Com essa avaliação é possivel aferir se a estratégia recomendada é significativa para o estudante e em paralelo se está, de fato, correlacionada com a sua motivação. Até o presente momento, os estudantes têm dado boas avaliações às estratégias recomendadas.

\section{Introdução}

Ambientes Virtuais de Aprendizagem (AVAs) coletam dados que podem ser úteis para definir o perfil de comportamento dos estudantes, além de identificar suas dificuldades e necessidades. É possível observar as ações que os estudantes executam dentro do sistema, sendo que essas formam caminhos que são conhecidos como Trilhas de Aprendizagem (TAs). Este estudo considera a TA como a trajetória percorrida pelo estudante, durante a sua interação com recursos e atividades disponibilizados no AVA. Em um trabalho anterior [Ramos et al. 2017], mostrou-se que a utilização das TAs foi capaz de auxiliar na análise do comportamento de estudantes de um ambiente virtual. 
Em paralelo, a Psicologia Educacional busca compreender o comportamento do estudante, por exemplo, com base em suas motivações e como tal comportamento influência na aprendizagem. A motivação é considerada como um constructo multidimensional e complexo [Bzuneck e Boruchovitch 2016], permitindo que possa ser estudado sob várias perspectivas. Uma das teorias da motivação que tem sido amplamente aceita é a Teoria de Metas de Realização [Ames 1984, Dweck 1986, Dweck e Elliott 1983], onde existem as orientações de metas.

A orientação de metas representa disposições e preferências pessoais que contribuem para a seleção de metas (objetivos estabelecidos). Ela pode ser definida como uma preferência de uma pessoa por classes específicas de estados finais desejados [Niemivirta 2002]. Diversos estudos [Elliot et al. 2011, Elliot e McGregor 2001, Elliot e Murayama 2008, Pulkka e Niemivirta 2013, Romero et al. 2019] têm mostrado ao longo dos anos que a Teoria de Metas de Realização pode ser utilizada, só para mencionar alguns exemplos, para compreender as estratégias de aprendizagem (EAs) adotadas pelo estudante, o desempenho (nota), a reação ao fracasso, o medo de falhar e a relação com os colegas de classe.

A identificação da orientação de metas, em sua maioria, é realizada por meio da aplicação de um questionário. Até o presente momento, apenas um trabalho [Romero et al. 2019] apresenta uma opção diferente, onde os autores propõem uma ferramenta computacional (programa de computador) onde o usuário executa algumas tarefas e, ao final, os dados gerados permitem identificar a sua orientação. Ainda não foram encontrados na literatura trabalhos que aproveitam as informações armazenadas nos AVAs, principalmente TAs, para classificar os estudantes segundo a Teoria de Metas de Realização.

A proposta deste trabalho é apresentar um framework que possibilita integrar as TAs com a identificação de motivação (orientação de metas) e as EAs. No framework proposto, coleta-se as TAs do estudante do ambiente que ele utilizar, e via questionário, coleta-se os dados para identificar a motivação. O módulo de classificação processa os dados do questionário e atribui a motivação ao modelo do estudante.

O módulo de recomendação utiliza os dados sobre a motivação do estudante, mais as EAs armazenadas. As estratégias estarão associadas às motivações. O docente, por meio de uma interface, visualiza as motivações e as estratégias disponíveis para cada tipo de motivação, e envia as recomendações aos estudantes. Os estudantes, por sua vez, recebem as estratégias e as avalia com estrelas. Com isso, é possível identificar se a estratégia recomendada é significativa para o estudante e, em paralelo, se é de fato correlacionada com a sua motivação.

\section{Trabalhos relacionados}

O trabalho apresentado por [Ramos et al. 2018] descreve um framework conceitual que usa os dados das TAs dos estudantes para recomendar agrupamentos de discentes para serem utilizados em atividades colaborativas em Ambientes Virtuais de Aprendizagem. O framework tem um módulo chamado M-Cluster que realiza a classificação usando os atributos extraídos das TAs com o uso do K-Means integrado às métricas de similaridades: distância Euclidiana, Manhattan e Cosseno. Cada classificação com uma métrica gera um resultado que pode ser visualizado pelo docente por meio do gráfico de bolhas ou visualização descritiva. 
A pesquisa de [Melo et al. 2017] consiste em um modelo do estudante baseado em perfil emocional (emoções e perfis de personalidade) para recomendação de estratégias pedagógicas personalizadas, denominado de Emotion-P para Ambientes Virtuais de Aprendizagem. Esse modelo utiliza a coleta de dados explícita por meio de questionários. Com os dados do modelo é possível escolher as estratégias pedagógicas que podem ser usadas para cada perfil de estudante.

O trabalho de agrupamento também utiliza TAs para modelo de estudante, entretanto as TAs não são categorizadas e a finalidade é a recomendação de grupos. Já o Emotion- $P$, requer uso de visão computacional para identificar emoções. Ele também recomenda estratégias, porém são ações pedagógicas ao docente. Como contribuição deste trabalho, propõe-se a classificação das TAs e o uso de um constructo relevante, mas que exige um custo menor para a identificação, no caso a motivação.

\section{Metodologia}

Inicialmente, foi criado o modelo do estudante unificando-se a TA e a motivação. Para as TAs, utilizou-se o modelo baseado em grafos proposto por [Ramos et al. 2017], onde foram criadas métricas como, por exemplo, a medida de dispersão. Também é possível utilizar medidas estatísticas como média, desvio padrão e variância sobre os dados de acesso dos vértices. Para complementar o modelo do estudante, identificou-se a orientação de meta sob a ótica da Teoria de Metas de Realização, por meio de um instrumento de medição validado denominado EMAPRE-U [Santos et al. 2013, Zenorini e Santos 2010].

De posse desse conjunto de informações e aplicando-se técnicas mineração de dados e aprendizagem de máquina [Artero, 2009], o passo seguinte foi encontrar padrões de TAs para cada meta de realização, criando-se um modelo de comportamento, com os quais também seja possível realizar predições de orientação de metas a partir de novas entradas de dados.

Em paralelo, foi elaborado um conjunto de sugestões de EAs a serem tomadas para associar aos modelos de comportamento. A associação de sugestões serve para auxiliar o estudante no uso de EAs mais adequadas ao seu perfil [Beluce e Oliveira 2016, Boruchovitch et al. 2006, Martins e Zerbini 2014]. Dessa forma, foi realizado um levantamento de diversos estudos sobre quais tipos de estratégias estão mais correlacionadas com cada tipo de meta de realização do instrumento selecionado, tais como [Beluce e Oliveira 2016, Costa et al. 2017, Perassinoto et al. 2013, Shyr et al. 2017].

Em seguida, foi desenvolvida uma ferramenta para validar o framework proposto, integrando o modelo de comportamento às EAs, onde as recomendações de estratégias puderam ser realizadas e avaliadas. Os detalhes do framework e da validação são apresentados nas seções seguintes.

\section{Arquitetura}

Nesta seção é apresentado o framework proposto que tem como objetivo recomendar EAs utilizando como modelo do estudante as TAs e as metas de realização (motivação). $O$ framework é composto pelo: modelo do estudante, que é composto pelas TAs e as orientações de metas de realização de cada estudante; EAs; módulo de classificação do estudante; e módulo de recomendação das EAs. 
No framework proposto, apresentado na Figura 1, a TA do estudante é coletada do ambiente que ele utilizar, e via questionário, são coletados os dados para calcular a motivação. As TAs e as motivações compõem o modelo do estudante. $\mathrm{O}$ módulo de classificação, então, calcula a motivação de acordo com a teoria adotada e atribui o resultado ao modelo do estudante. O módulo de recomendação utiliza os dados sobre a motivação do estudante, mais as EAs registradas no sistema. As estratégias sempre estarão associadas às motivações. O docente, por meio de uma interface, visualiza as motivações e as estratégias disponíveis para cada tipo de meta, e as sugere aos estudantes pelo próprio sistema. Os estudantes recebem as estratégias, no caso via e-mail, e as avalia com estrelas. Com isso, é verificado se a estratégia recomendada é significativa para o estudante, e em paralelo, é avaliado se de fato ela está correlacionada com a sua motivação.

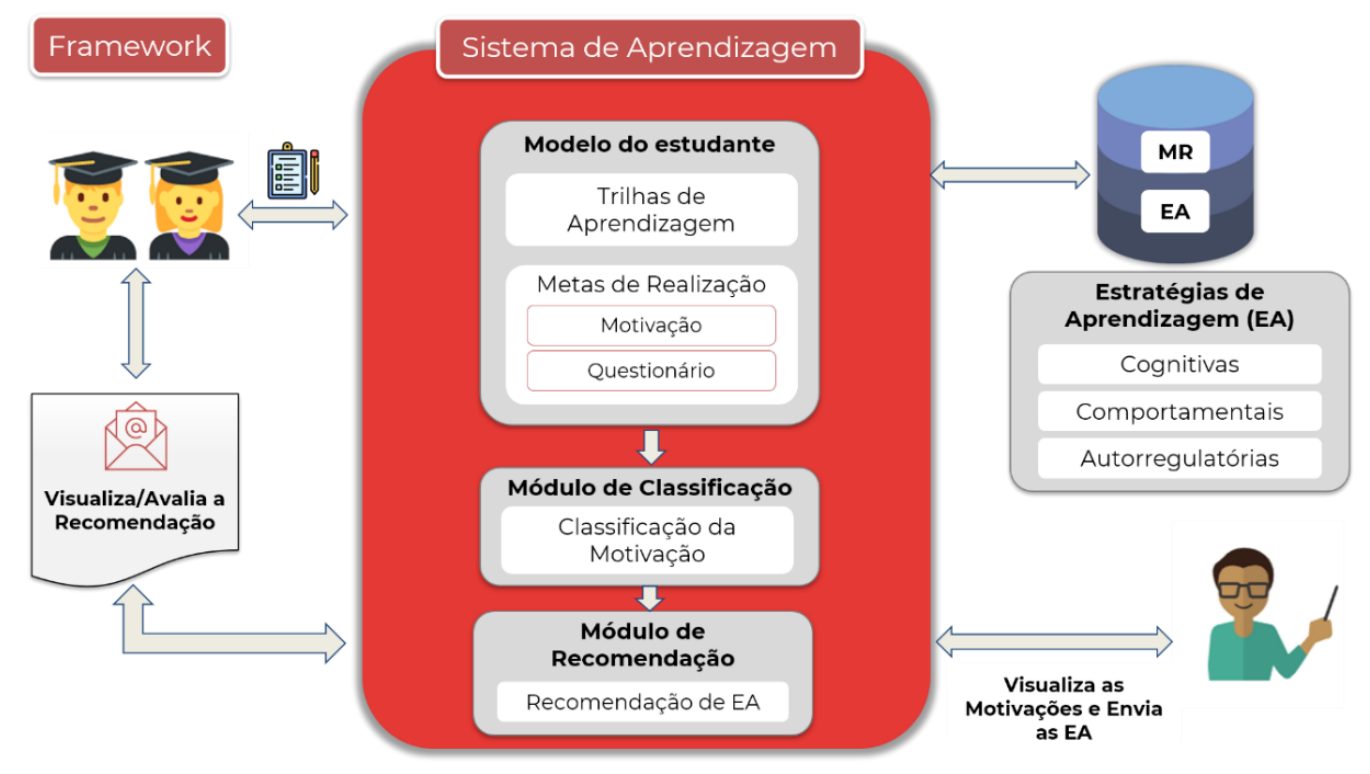

Figura 1. Arquitetura do Framework

A seguir, cada parte da arquitetura é descrita em detalhes: interface estudante, banco de dados, TAs, classificação da motivação, estratégias de aprendizagem e interface docente/tutor.

\subsection{Interface Estudante}

$\mathrm{O}$ estudante interage com o sistema de três formas, todas complementares. A primeira forma é responder um formulário de escala de motivação no sistema de aprendizagem. A escala de motivação, no contexto da educação, é um instrumento desenvolvido na área da psicologia educacional que é testado e validado para comprovar as suas propriedades psicométricas. No caso deste trabalho, optou-se por utilizar uma escala de motivação baseada na Teoria de Metas de Realização.

Ainda por meio do sistema de aprendizagem, o estudante interage com os materiais e atividades disponíveis. Os dados dessa interação são coletados automaticamente pelo sistema e, a partir deles, são identificadas as TAs do estudante. Essa coleta de dados se dá de forma transparente, ou seja, nada é perguntado ao estudante, basta ele utilizar o sistema de aprendizagem normalmente. 


\subsection{Banco de Dados}

Este framework utiliza as informações armazenadas no banco de dados dos sistemas de aprendizagem, além do conjunto de dados adicionais relativos à motivação e EAs. Os dados adicionais são independentes do sistema de aprendizagem, tornando a abordagem proposta flexível. Isso permite que o framework seja adaptado com maior facilidade a contextos e abordagens diferentes.

As principais informações coletadas do banco de dados foram estabelecidas para se obter a TA proposta por [Ramos et al. 2017]. As TAs irão compor o modelo do estudante, juntamente com a motivação.

Enquanto as TAs são obtidas de forma automatizada, ou seja, sem interação direta com o estudante, as motivações são obtidas de forma manual via questionário. $\mathrm{O}$ questionário pode ser respondido pelo estudante dentro do sistema de aprendizagem utilizado ou externamente, a depender de como se dá a integração do framework com o sistema de aprendizagem. Em ambos os casos, as respostas são armazenadas no banco de dados para que sejam usadas para calcular as orientações de motivações dos estudantes.

Outro elemento fundamental do framework são as EAs. Elas são armazenadas em um banco de dados do framework. A seguir são apresentados mais detalhes das TAs, motivação e EAs.

\subsection{Trilhas de Aprendizagem}

As TAs representam a interação do usuário com o sistema de aprendizagem, em específico com os recursos e atividades que compõe o curso do estudante. Por meio das trilhas é possível realizar um acompanhamento do estudante usando-se apenas as informações do sistema de aprendizagem. $\mathrm{O}$ framework proposto trabalha com as TAs como definindo por [Ramos et al. 2017], que são representadas na forma de grafos, conforme a Figura 2.

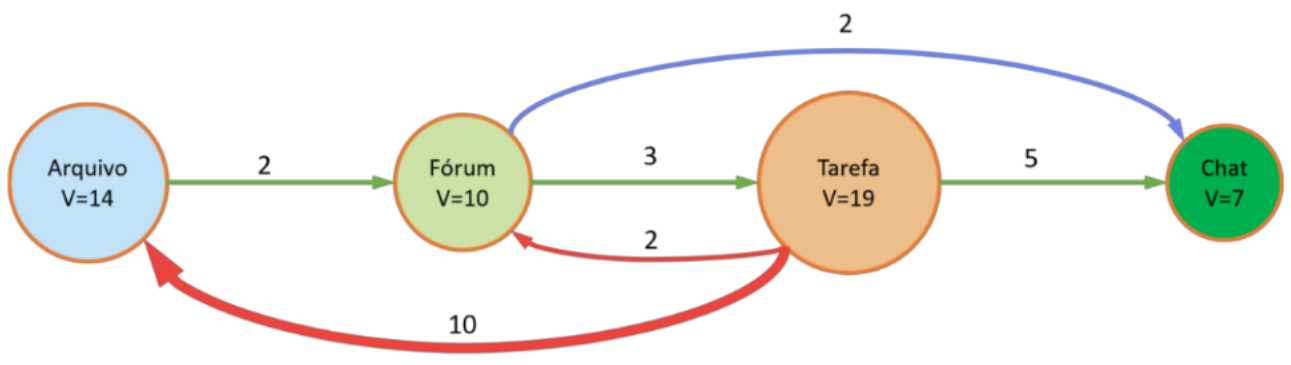

Figura 2. Trilha de aprendizagem. Fonte: [Ramos et al. 2017]

O principal destaque dessa abordagem é que foram definidos três tipos de arestas e uma métrica chamada dispersão. As arestas são classificadas em avanço, retorno e padrão e estabelecem o sentido que o estudante percorre sua TA, se avançando, voltando e seguindo sequencialmente os recursos e atividades, estes são representados por vértices. Já a métrica dispersão foi criada com o intuito de medir o quão dispersa é a trajetória do estudante.

\subsection{Classificação da Motivação}

A motivação do estudante implica diretamente no processo de aprendizagem [Santos et al. 2013], sendo considerado um dos principais fatores da promoção da aprendizagem 
[Zenorini e Santos 2010]. Dessa forma, a motivação irá compor o modelo do estudante juntamente com as TAs. Escolheu-se dentre as teorias utilizadas para o estudo da motivação a Teoria de Metas de Realização, pois representa o motivo pelo qual uma pessoa executa uma tarefa [Santos et al. 2013] .

A metas variam de acordo com as pesquisas, bem como os instrumentos que as medem, que em sua grande maioria são questionários. Ao selecionar um instrumento, $\mathrm{o}$ mais importante é que ele seja na língua do respondente e devidamente validado. A motivação dos estudantes é calculada a partir do instrumento respondido. Uma vez identificado a motivação/orientação da meta, ela é armazenada no banco de dados e compõe o modelo do estudante. Dessa forma, é possível investigar a relação entre as TAs e a motivação do estudante.

O SisREA (Sistema de Recomendação de Estratégias de Aprendizagem) foi a ferramenta criada a partir do framework. Para compor o SisREA foi selecionado o instrumento chamado EMAPRE-U [Santos et al. 2013, Zenorini e Santos 2010], para identificar a motivação de aprendizagem de cada participante e utilizar essa informação para classificar a sua TA. O instrumento EMAPRE-U, disponível em [Ramos 2020], é um instrumento validado ao contexto universitário em língua portuguesa, devidamente aprovado pela avaliação de suas propriedades psicométricas [Santos et al. 2013]. O instrumento possui 28 itens, divididos em três metas de realização: 12 itens para a meta aprender, 9 para a meta performance aproximação e 7 itens para a meta performance evitação. O instrumento é respondido em uma escala de Likert de três pontos: "Discordo", "Não sei" e "Concordo".

\subsection{Estratégias de Aprendizagem}

Uma vez que a motivação para aprender do estudante seja identificada, o próximo passo é encontrar uma finalidade para essa informação. Neste trabalho, pensamos em colaborar com o estudante no sentido de lhe apresentar as EAs correlacionadas com a sua orientação de meta. A adoção de EAs é importante para transpor dificuldades e permitir maior sucesso no aprendizado, como por exemplo, ao ensiná-las a estudantes de baixo rendimento escolar [Boruchovitch et al. 2006]. É possível que o alinhamento das metas de realização e as EAs possa melhorar a aprendizagem do estudante.

As EAs criadas, disponíveis em [Ramos 2020], foram extraídas e adaptadas das escalas validadas de EAs de alguns trabalhos da área [Beluce e Oliveira 2016, Boruchovitch et al. 2006, Boruchovitch e Santos 2015, Martins e Zerbini 2014], que abordam EAs aplicados a estudantes brasileiros, garantindo que as estratégias recomendadas estejam de acordo com os trabalhos na literatura. As EAs então são associadas a uma ou mais orientações de meta. Dessa forma, quando se identifica a meta do estudante é possível saber quais EAs são adequadas a ela.

\subsection{Interface Docente/Tutor}

$\mathrm{Na}$ interface do tutor são exibidos: os estudantes, as suas motivações já classificadas, as estratégias registradas no sistema e as opções de recomendação de EAs. Por essa interface, o docente consegue visualizar as turmas e os estudantes que responderam ao questionário, e que, portanto, possuem a motivação classificada. O docente também consegue visualizar quais estratégias já foram recomendadas para cada estudante, assim como as notas que eles deram, caso já as tenham avaliado. 


\section{Validação Inicial e Resultados}

O SisREA foi aplicado junto a um sistema de aprendizagem, no formato de juiz online [CodeBench 2020], no qual os estudantes voluntários, de cursos superiores de diversas áreas, aprendem conceitos de programação, nos semestres 2019/2 (152 estudantes) e 2020/1 (152 estudantes), ao todo 304 estudantes. Ao utilizar o ambiente de aprendizagem, as ações dos estudantes são armazenadas nos $\log s$, de onde são extraídas as informações para a composição das TAs, as principais informações são o acesso ao material didático e às listas de tarefas (exercícios de programação), que são respondidas no próprio ambiente. O modelo das TAs [Ramos et al. 2017] ainda está sendo adaptado ao juiz online, assim ainda não foi possível trabalhar a classificação das metas de realização a partir das TAs.

Integrado ao ambiente, os estudantes responderam ao EMAPRE-U. As respostas foram armazenadas no banco de dados do sistema de aprendizagem. O módulo de classificação do SisREA acessa as respostas e calcula a orientação de meta do estudante e armazena a resposta no SisREA. Prevalece a meta que tiver maior índice.

Com os estudantes já classificados, o docente pode acessar o SisREA para fazer as recomendações de EAs. Para isso ele, primeiro seleciona a turma, e pressiona o botão 'Recomendar'. Em seguida, escolhe, a seu critério, a estratégia para cada estudante, a partir da lista pré-estabelecida que contém apenas as estratégias relacionadas à motivação do estudante, e clica em 'Enviar Recomendação', conforme a Figura 3.

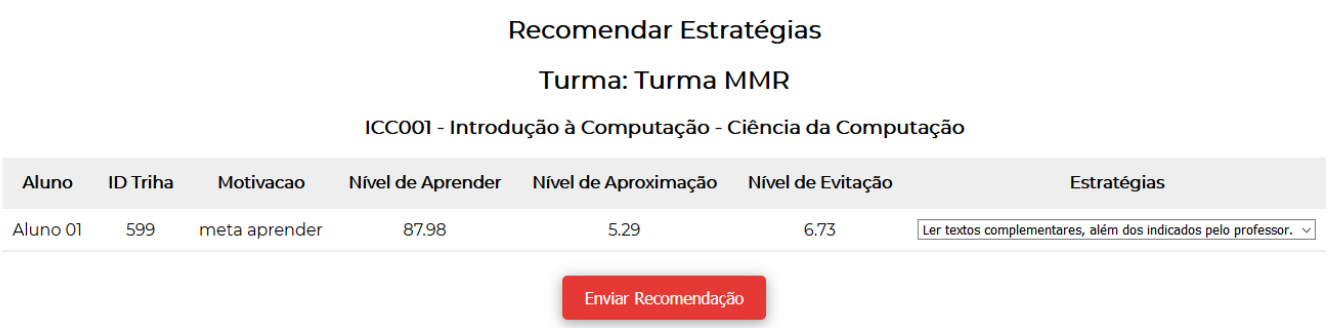

Figura 3. Enviando recomendação para uma turma.

O estudante recebe a recomendação em seu e-mail, na qual aparecem a EA e uma classificação na escala de Likert, em formato cinco estrelas, conforme a Figura 4. As avaliações dos estudantes ficam então armazenadas e podem ser utilizadas para identificar as melhores recomendações. Os resultados podem ser considerados promissores, pois, em sua maioria, os estudantes têm avaliado as estratégias com 4 ou 5 estrelas. Apenas os alunos de 2019/2 avaliaram as EAs, uma vez que a pandemia paralisou as aulas em 2020.

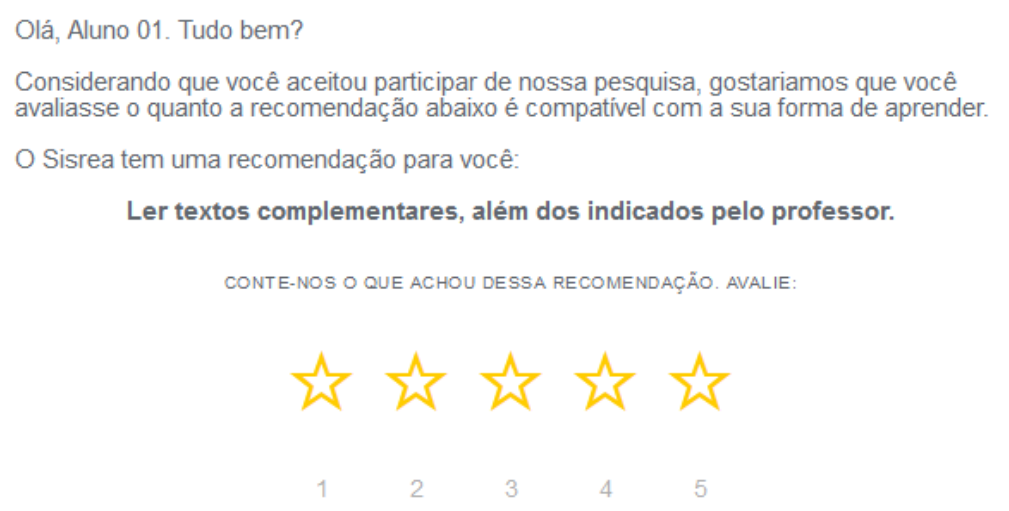

Figura 4. E-mail recebido pelo estudante. 


\section{Conclusões e Trabalhos Futuros}

Estou trabalho apresentou um framework conceitual para a recomendação de EAs, visando promover o conhecimento e uso de EAs por parte dos estudantes. O framework também proporciona ao docente conhecer a orientação de meta de seus estudantes, bem com auxiliou na sugestão de EAs mais apropriadas com a motivação do estudante. Para se chegar ao desenvolvimento do framework, foi realizada uma pesquisa sobre motivação, sob a perspectiva da Teoria de Metas de Realização e sobre EAs.

A arquitetura geral do framework foi projetada pensando na flexibilidade, tais como a utilização de outros instrumentos que avaliam as metas de realização, bem como inclusão de novas EAs. Como contribuições do trabalho, pode-se citar: a recomendação das EAs alinhadas com as orientações de metas de realização do estudante; a criação de um framework que organiza o processo de identificação das TAs, das metas de realização e sugestão das EAs; e o desenvolvimento de um sistema que pode ser integrado ao sistemas de aprendizagem já existentes.

Uma ferramenta foi desenvolvida a partir do framework, e foi aplicada em turmas do ensino superior em disciplinas de programação. A ferramenta conseguiu identificar as metas de realização, por meio de um instrumento aplicado de forma integrada ao sistema de aprendizagem, o que permitiu realizar as recomendações de acordo com a motivação de cada estudante. Como limitação do trabalho a extração das TAs depende fortemente do sistema de aprendizagem utilizado, o que exige uma implementação específica.

Para os trabalhos futuros pretende-se: modelar a relação entre as TAs e as metas de realização, de forma automática, por meio de técnicas de aprendizagem de máquina e mineração de dados; integrar o framework com o AVA Moodle; realizar o ranqueamento das EAs utilizando a avaliação da estratégia realizada pelo estudante, para auxiliar na escolha das estratégias.

\section{Agradecimentos}

O presente trabalho foi realizado com apoio da Coordenação de Aperfeiçoamento de Pessoal de Nível Superior - Brasil (CAPES) - Código de Financiamento 001 e do Instituto Federal de Educação, Ciência e Tecnologia do Estado do Amazonas - IFAM Campus Parintins. O primeiro autor também agradece a Fundação de Amparo à Pesquisa do Estado do Amazonas (FAPEAM) pela bolsa de pesquisa concedida por meio do Programa PROINT/AM (Edital Nº03/2018).

\section{Referências}

Ames, C. (1984). Competitive, cooperative and individualistic goal structures: A motivational analysis. Research on Motivation in Education. Vol. 1: Student Motivation, 1, 177-207.

Artero, A. O. (2009). Inteligência Artificial: Teoria e Prática (1st ed.). Livraria da Física.

Beluce, A. C., \& Oliveira, K. L. de. (2016). Escala de estratégias e motivação para aprendizagem em ambientes virtuais. Revista Brasileira de Educação, 21(66), 593610. https://doi.org/10.1590/S1413-24782016216631

Boruchovitch, E., Santos, A. A. A. dos, da Costa, E. R., Neves, E. R. C., Cruvinel, M., Primi, R., \& Guimarães, S. E. R. (2006). A construção de uma escala de estratégias 
IX Congresso Brasileiro de Informática na Educação (CBIE 2020)

Anais do XXXI Simpósio Brasileiro de Informática na Educação (SBIE 2020)

de aprendizagem para alunos do ensino fundamental. Psicologia: Teoria e Pesquisa, 22(3), 297-304.

Boruchovitch, Evely, \& Dos Santos, A. A. A. (2015). Psychometric studies of the learning strategies scale for university students. Paideia, 25(60), 19-27. https://doi.org/10.1590/1982-43272560201504

Bzuneck, J. A., \& Boruchovitch, E. (2016). Motivação e Autorregulação da Motivação no Contexto Educativo. Psicologia: Ensino \& Formação, 7(2), 75-84. https://doi.org/10.21826/2179-58002016727584

CodeBench. (2020). CodeBench. http://codebench.icomp.ufam.edu.br/

Costa, M. A. S., Silva, B. N. da, \& Abbas, K. (2017). Motivações e estratégias de aprendizagem dos discentes em contabilidade de custos e a influência no desempenho acadêmico. XXIV Congresso Brasileiro de Custos.

Dweck, C. S. (1986). Motivational Processes Affecting Learning. American Psychologist. https://doi.org/10.1037/0003-066X.41.10.1040

Dweck, C. S., \& Elliott, E. S. (1983). Achievement motivation. In E. M. Hetherington (Ed.), Handbook of child psychology: Socialization, personality, and social development (pp. 643-691). Wiley.

Elliot, A. J., \& McGregor, H. A. (2001). A 2 x 2 achievement goal framework. Journal of Personality and Social Psychology, 80(3), 501-519. https://doi.org/10.1037/0022-3514.80.3.501

Elliot, A. J., \& Murayama, K. (2008). On the Measurement of Achievement Goals: Critique, Illustration, and Application. Journal of Educational Psychology, 100(3), 613-628. https://doi.org/10.1037/0022-0663.100.3.613

Elliot, A. J., Murayama, K., \& Pekrun, R. (2011). A $3 \times 2$ achievement goal model. Journal of Educational Psychology, 103(3), 632-648. https://doi.org/10.1037/a0023952

Elliot Andrew J., \& Church Marcy A. (1997). A hierarchical model of approach and avoidance achievement motivation. Journal of Personality and Social Psychology, 72(1), 218-232.

Harackiewicz, J. M., Barron, K. E., Tauer, J. M., Carter, S. M., \& Elliot, A. J. (2000). Short-term and long-term consequences of achievement goals. Journal of Educational Psychology, 92(2), 316-330.

Hayamizu, T., \& Weiner, B. (1991). A Test of Dweck's Model of Achievement Goals as Related to Perceptions of Ability. The Journal of Experimental Education, 59(3), 226-234. https://doi.org/10.1080/00220973.1991.10806562

Martins, L. B., \& Zerbini, T. (2014). Escala de Estratégias de Aprendizagem: evidências de validade em contexto universitário híbrido. Psico-USF, 19(2), 317-328. https://doi.org/10.1590/1413-82712014019002007

Melo, S. De, Dantas, A. C., \& Fernandes, M. (2017). Modelo do estudante baseado em emoções e perfis de personalidade para recomendação de estratégias pedagógicas personalizadas. Anais Do XXVIII Simpósio Brasileiro de Informática Na Educação (SBIE 2017), 1(Cbie), 967. https://doi.org/10.5753/cbie.sbie.2017.967 
Niemivirta, M. (2002). Motivation and performance in context: The influence of goal orientations and instructional setting on situational appraisals and task performance. PSYCHOLOGIA -An International Journal of Psychology in the Orient, 45(4), 250270. https://doi.org/10.2117/psysoc.2002.250

Perassinoto, M., Boruchovitch, E., \& Bzuneck, J. (2013). Estratégias de aprendizagem e motivação para aprender de alunos do Ensino Fundamental. Avaliaçao Psicologica: Interamerican Journal of Psychological Assessment, 12(3), 351-359.

Pulkka, A., \& Niemivirta, M. (2013). Predictive relationships between adult students ' achievement goal orientations, course evaluations, and performance. International Journal of Educational Research, 61, 26-37. https://doi.org/10.1016/j.ijer.2013.03.015

Ramos, D. B. (2020). Motivação e Estratégias de Aprendizagem. 2020. http://mobmoodle.icomp.ufam.edu.br/projeto/?page_id=159

Ramos, D., Monteverde, I., Nascimento, P. Do, Amaral, G., \& Oliveira, E. (2017). Um modelo para Trilhas de Aprendizagem em um Ambiente Virtual de Aprendizagem. Simpósio Brasileiro de Informática Na Educação (SBIE), Cbie, 1407. https://doi.org/10.5753/cbie.sbie.2017.1407

Ramos, I. M. M., Ramos, D. B., Amaral, G. de S., Gadelha, B. F., \& Oliveira, E. H. T. de. (2018). Framework Conceitual para Formação de Grupos de Alunos utilizando Trilhas de Aprendizagem em um Ambiente Virtual de Aprendizagem. Simpósio Brasileiro de Informática Na Educação (SBIE 2018), 1673-162. https://doi.org/10.5753/cbie.sbie.2018.1673

Romero, M., Hernández, J. M., Juola, J. F., Casadevante, C., \& Santacreu, J. (2019). Goal Orientation Test: An Objective Behavioral Test. Psychological Reports, 003329411984584. https://doi.org/10.1177/0033294119845847

Santos, A. A. A. dos, Alcará, A. R., \& Zenorini, R. da P. C. (2013). Estudos psicométricos da escala de motivação para a aprendizagem de universitários. Fractal : Revista de Psicologia, 25(3), 531-546. https://doi.org/10.1590/s1984-02922013000300008

Shyr, W.-J., Feng, H.-Y., Zeng, L.-W., Hsieh, Y.-M., \& Shih, C.-Y. (2017). The Relationship between Language Learning Strategies and Achievement Goal Orientations from Taiwanese Engineering Students in EFL Learning. EURASIA Journal of Mathematics, Science and Technology Education, 13(10), 6431-6443. https://doi.org/10.12973/ejmste/76660

Zenorini, R. da P. C., \& Santos, A. A. A. dos. (2010). Escala de Metas de Realização como Medida da Motivação para Aprendizagem. Interamerican Journal of Psychology, 44(2), 291-298. 